\title{
Pointwise upper estimates for transition probabilities of continuous time random walks on graphs
}

\author{
Xinxing Chen \\ Department of Mathematics, Shanghai Jiaotong University, Shanghai 200240,China.E-mail: chenxinx@sjtu.edu.cn
}

Received 22 April 2014; revised 21 July 2015; accepted 31 July 2015

\begin{abstract}
Let $X$ be a continuous time random walk on a weighted graph. Given the on-diagonal upper bounds of transition probabilities at two vertices $x_{1}$ and $x_{2}$, we obtain Gaussian upper estimates for the off-diagonal transition probability $\mathbb{P}_{x_{1}}\left(X_{t}=x_{2}\right)$ in terms of an adapted metric introduced by Davies.
\end{abstract}

Résumé. Soit $X$ une marche aléatoire à temps continu sur un graphe pondéré. Etant données des bornes supérieures sur la transition de probabilité diagonale en deux sommets $x_{1}$ et $x_{2}$, nous obtenons des estimées supérieures gaussiennes sur la transition de probabilité $\mathbb{P}_{x_{1}}\left(X_{t}=x_{2}\right)$ (qui est en dehors de la diagonale) en termes d'une métrique adaptée introduite par Davies.

MSC: 60G50; 30K08

Keywords: Random walk; Transition probability; Heat kernel; Gaussian upper bound

\section{Introduction}

Let $\Gamma=(\mathcal{V}, \mathbb{E})$ be a connected, locally finite graph without double edges. The graph $\Gamma$ can be either finite or infinite. Let $\mu$ be an edge weight function on $\mathbb{E}$, such that $\mu_{x y}=\mu_{y x}>0$ for each $(x, y) \in \mathbb{E}$, while $\mu_{x y}=0$ for each $(x, y) \notin \mathbb{E}$. Let $v_{x}>0$ for $x \in \mathcal{V}$. Denote by $X=\left\{X_{t}: t \geq 0\right\}$ a continuous time random walk on $\Gamma$ with generator

$$
\mathcal{L} f(x)=\frac{1}{v_{x}} \sum_{y \in \mathcal{V}}(f(y)-f(x)) \mu_{x y} .
$$

Write $\mathbb{P}_{x}$ for the probability measure of $X$ starting from $x$.

If $v_{x}=\sum \mu_{x y}$ for all $x$, then the process $X$ is called the constant speed random walk or CSRW on $\mathcal{V}$. It is a process that waits an exponential time mean 1 at each vertex and then jumps to one of its neighbours. If $v_{x} \equiv 1$, then the expected waiting time of each jump may vary. Moreover, such a process may explode in finite time.

In this paper, we fix vertices $x_{1}, x_{2} \in \mathcal{V}$ and functions $f_{1}, f_{2}$ on $\mathbb{R}_{+}$such that for any $i=1,2$ and $t \geq 0$,

$$
\mathbb{P}_{x_{i}}\left(X_{t}=x_{i}\right) \leq \frac{1}{f_{i}(t)}
$$

Our interest is, under what circumstances $\mathbb{P}_{x_{1}}\left(X_{t}=x_{2}\right)$ will have Gaussian upper bounds. Let $d_{v}(\cdot, \cdot)$ be a metric of $\Gamma$ such that

$$
\begin{cases}\frac{1}{v_{x}} \sum_{y} d_{v}(x, y)^{2} \mu_{x y} \leq 1 & \text { for all } x \in \mathcal{V}, \\ d_{v}(x, y) \leq 1 & \text { whenever } x, y \in \mathcal{V} \text { and } x \sim y .\end{cases}
$$


Metrics satisfying (1.2) are called adapted metrics. Such metrics were introduced by Davies [9] and [10], and are closely related to the intrinsic metric associated with a given Dirichlet form. (One might expect that analogues of diffusion processes on manifolds hold using the intrinsic metrics for random walks on graphs, see [12,13] and [17].) Fix $A \geq 1$ and $\gamma>1$. Let $f: \mathbb{R}_{+} \rightarrow \mathbb{R}_{+}$. We say that $f$ is $(A, \gamma)$-regular on $[a, b)$, if the function $f$ is non-decreasing on $\mathbb{R}_{+}$and satisfies that

$$
\frac{f(\gamma s)}{f(s)} \leq A \frac{f(\gamma t)}{f(t)} \text { for all } a \leq s<t<\gamma^{-1} b .
$$

In particular, if $a=0$ and $b=\infty$ then we say that $f$ is $(A, \gamma)$-regular, which was introduced by Grigor'yan [14].

Theorem 1.1. Let $\delta \geq 1$. If each $f_{i}$ is $(A, \gamma)$-regular and satisfies

$$
f_{i}(t) \leq A e^{\delta t} \quad \text { for all } t \in \mathbb{R}_{+},
$$

then there exist constants $C_{1}, \theta>0$ which are independent of $A, \gamma$ and $\delta$, such that

$$
\mathbb{P}_{x_{1}}\left(X_{t}=x_{2}\right) \leq \frac{C_{1} A^{\beta}\left(v_{x_{2}} / v_{x_{1}}\right)^{1 / 2}}{\sqrt{f_{1}(\alpha t) f_{2}(\alpha t)}} \exp \left(-\theta \frac{d_{v}\left(x_{1}, x_{2}\right)^{2}}{t}\right) \quad \text { for } t \geq d_{\nu}\left(x_{1}, x_{2}\right),
$$

where $\alpha=\min \left\{(2 \gamma)^{-1},(64 \delta)^{-1}\right\}$ and $\beta=\left\lceil\frac{\log \gamma}{\log 2}\right\rceil$.

The problem of getting a Gaussian upper bound from two point estimates was introduced in the manifold case by Grigor'yan [14]. In subsequent researches, Coulhon, Grigor'yan and Zucca [8] studied the problem for discrete time random walks on graphs, while Folz [11] studied it for the continuous time random walks. The current paper considers the same problem, however, it improves the result of [11] by no longer requiring a lower bound on $v_{x}$. The improvement comes from imposing conditions on the transition probabilities $\mathbb{P}_{x}\left(X_{t}=x\right)$ instead of the heat kernels $p_{t}(x, x)$. Note that the transition probabilities are invariant under the transformation from $(\mu, v)$ to $(c \mu, c \nu)$, where $(c \mu)_{x y}=c \mu_{x y}$ and $(c \nu)_{x}=c v_{x}$.

Remark 1.1. The condition (1.4) is quite natural. Note that $\mathbb{P}_{x}\left(X_{t}=x\right) \geq \exp \left(-\frac{\mu_{x}}{v_{x}} t\right)$, where $\mu_{x}=\sum_{y} \mu_{x y}$. It implies that (1.4) holds if $A=1$ and $\delta=\max \left\{\frac{\mu_{x_{1}}}{v_{x_{1}}}, \frac{\mu_{x_{2}}}{v_{x_{2}}}\right\}$. In particular, for CSRW one can take $\delta=1$.

Remark 1.2. One can also trace the values of $C_{1}$ and $\theta$. Indeed, we select $\theta=10^{-7}$ in our proof.

Theorem 1.2. Let $\delta \geq 1$. If each $f_{i}$ is $(A, \gamma)$-regular on $\left[T_{1}, T_{2}\right)$ and satisfies

$$
f_{i}(t) \leq A e^{\delta t} \text { for all } t \in\left[T_{1}, T_{2}\right)
$$

then there exist constants $C_{1}, \theta>0$ which are independent of $A, \gamma$ and $\delta$, such that

$$
\mathbb{P}_{x_{1}}\left(X_{t}=x_{2}\right) \leq \frac{C_{1} A^{\beta}\left(v_{x_{2}} / v_{x_{1}}\right)^{1 / 2}}{\sqrt{f_{1}(\alpha t) f_{2}(\alpha t)}} \exp \left(-\theta \frac{d_{v}\left(x_{1}, x_{2}\right)^{2}}{t}\right) \quad \text { for } t \in\left[\widetilde{T}_{1}, T_{2}\right),
$$

where $\alpha=\min \left\{(2 \gamma)^{-1},(64 \delta)^{-1}\right\}, \beta=\left\lceil\frac{\log \gamma}{\log 2}\right\rceil$ and $\widetilde{T}_{1}=\left(8 \alpha^{-2} T_{1}^{2}\right) \vee d_{v}\left(x_{1}, x_{2}\right)$.

\section{Remark 1.3.}

(1) If the growth rate of $f_{i}$ is either sub-exponential or polynomial, then the lower bound of $\widetilde{T}_{1}$ will be improved, see Theorems 5.1 and 5.2. 
(2) Theorems 1.1 and 1.2 are potentially very useful for random walks with random conductances. For example, Mathieu and Remy [16] considered the CSRW on the infinite cluster $\mathcal{C}_{\infty}(\omega)$ and showed that $\mathbb{P}_{x}\left(X_{t}=x\right) \leq c t^{-d / 2}$ for $t \geq N_{x}(\omega)$ and $x \in \mathcal{C}_{\infty}(\omega)$. Using Theorem 1.2 immediately gives

$$
\mathbb{P}_{x}\left(X_{t}=y\right) \leq c_{1} t^{-d / 2} \exp \left(-c_{2} \frac{d(x, y)^{2}}{t}\right), \quad t \geq S_{x y}(\omega) \vee d(x, y),
$$

where $S_{x y}(\omega)=64^{3}\left(N_{x}(\omega)^{2} \vee N_{y}(\omega)^{2}\right)$ and $d(x, y)$ is the graph distance. A more delicate result by a different method was obtained in [2].

See Balow and Chen [3] for the new application in a deterministic graph where volume doubling and Poincaré inequality hold for all sufficiently large balls.

In Section 2, we show the Integral Maximum Principle for a positive subsolution function on $\mathbb{R}_{+} \times \mathcal{V}$. From this, we get the initial estimates of the transition probabilities, the case $t \leq d_{v}(x, y)$ included. In Section 3 , we update the results of the previous section, under the assumption that a certain regularity condition holds. In Section 4, we give the proof of Theorem 1.1. In the final section, we consider functions which are regular only on an interval and have different rates of growth; in doing so, we obtain Theorem 1.2.

\section{Integral maximum principle}

For any functions $f, g$ on $\mathcal{V}$, define

$$
\langle f, g\rangle=\sum_{x \in \mathcal{V}} f(x) g(x) v_{x} .
$$

Then $\langle\cdot, \cdot\rangle$ induces an inner product space. Denote by $\|\cdot\|$ the induced norm. Let $\mathbb{I}$ be an interval of $\mathbb{R}_{+}$. We say that $u: \mathbb{I} \times \mathcal{V} \mapsto \mathbb{R}_{+}$is a positive subsolution of the heat equation on $\mathbb{I} \times \mathcal{V}$ if

$$
\frac{\partial}{\partial t} u \leq \mathcal{L} u \quad \text { on } \mathbb{I} \times \mathcal{V} .
$$

Furthermore, we define a set of functions:

$$
\mathcal{H}(\mathbb{I})=\{u: u \text { is a positive subsolution on } \mathbb{I} \times \mathcal{V} \text { and } \mid\{z: u(t, z) \neq 0 \text { for some } t \in \mathbb{I}\} \mid<\infty\} \text {. }
$$

Let $o \in B \subseteq \mathcal{V}$ with $|B|<\infty$. Set

$$
u_{B}(t, z)=\frac{v_{o}^{1 / 2}}{v_{z}} \mathbb{P}_{o}\left(X_{t}=z, \inf \left\{s \geq 0: X_{s} \notin B\right\}>t\right) .
$$

Then $u_{B}=0$ on $\mathbb{R}_{+} \times(\mathcal{V} \backslash B)$. Since $\Gamma$ is a locally finite graph, $u_{B}$ is a positive subsolution on $\mathbb{R}_{+} \times \mathcal{V}$ and so $u_{B} \in \mathcal{H}\left(\mathbb{R}_{+}\right)$. Now we show the Integral Maximum Principle.

Theorem 2.1. Let $h$ be a positive function on $\mathbb{I} \times \mathcal{V}$ and $u \in \mathcal{H}(\mathbb{I})$. If for each $t \in \mathbb{I}$ one has

$$
\frac{1}{v_{y}} \sum_{x} \frac{|h(t, x)-h(t, y)|^{2}}{4 h(t, x) h(t, y)} \mu_{x y} \leq-\frac{\partial}{\partial t} \log h(t, y) \quad \text { for all } y \in \mathcal{V},
$$

then $J(t)=\left\langle u^{2}(t, \cdot), h(t, \cdot)\right\rangle$ is non-increasing on $\mathbb{I}$. 
Proof. For brevity, we omit the notation $t$. Set $\nabla_{x y} g=g(t, y)-g(t, x)$ for any function $g$ on $\mathbb{I} \times \mathcal{V}$ and get

$$
\begin{aligned}
\langle 2 u \mathcal{L} u, h\rangle & =2\langle u h, \mathcal{L} u\rangle \\
& =-\sum_{x, y} \nabla_{x y}(u h) \cdot \nabla_{x y} u \cdot \mu_{x y} \quad \text { since } \mid\{z: u(t, z) \neq 0 \text { for some } t \in \mathbb{I}\} \mid<\infty \\
& =-\sum_{x, y}\left(h(x) \nabla_{x y} u+u(y) \nabla_{x y} h\right) \cdot \nabla_{x y} u \cdot \mu_{x y} \\
& =-\sum_{x, y}\left(\left(\nabla_{x y} u\right)^{2} h(x)+u(y) \nabla_{x y} u \cdot \nabla_{x y} h\right) \mu_{x y} \\
& =\sum_{x, y}\left[-\left(\sqrt{h(x)} \nabla_{x y} u+\frac{u(y) \nabla_{x y} h}{2 \sqrt{h(x)}}\right)^{2}+\frac{\left(u(y) \nabla_{x y} h\right)^{2}}{4 h(x)}\right] \mu_{x y} \quad \text { since } h \text { is positive } \\
& \leq \sum_{x, y} u(y)^{2} \frac{\left|\nabla_{x y} h\right|^{2}}{4 h(x)} \mu_{x y} \\
& =\sum_{y} u(y)^{2}\left(\sum_{x} \frac{\left|\nabla_{x y} h\right|^{2}}{4 h(x)} \mu_{x y}\right) .
\end{aligned}
$$

By (2.2), $\sum_{x} \frac{\left|\nabla_{x y} h\right|^{2}}{4 h(x)} \mu_{x y} \leq-v_{y} \frac{\partial}{\partial t} h(y)$ and hence

$$
\langle 2 u \mathcal{L} u, h\rangle \leq-\sum_{y} u(y)^{2} v_{y} \frac{\partial}{\partial t} h(y)=-\left\langle u^{2}, \frac{\partial}{\partial t} h\right\rangle .
$$

On the other hand, by the condition that $u$ is a positive subsolution on $\mathbb{I} \times \mathcal{V}$, we have

$$
\frac{d}{d t} J=\frac{\partial}{\partial t}\left\langle u^{2}, h\right\rangle=\left\langle 2 u \frac{\partial}{\partial t} u, h\right\rangle+\left\langle u^{2}, \frac{\partial}{\partial t} h\right\rangle \leq\langle 2 u \mathcal{L} u, h\rangle+\left\langle u^{2}, \frac{\partial}{\partial t} h\right\rangle \leq 0 .
$$

Therefore, $J$ is non-increasing.

Since the metric $d_{v}$ satisfies (1.2), Theorem 2.1 immediately implies Corollary 2.2 as follows. Define a set of functions:

$$
\begin{aligned}
\mathcal{F}(\mathbb{I})= & \{h: h \text { is a positive function on } \mathbb{I} \times \mathcal{V} \text { and for each } t \in \mathbb{I}, x, y \in \mathcal{V} \text { with } x \sim y, \\
& \left.\frac{|h(t, x)-h(t, y)|^{2}}{4 h(t, x) h(t, y)} \leq-d_{v}(x, y)^{2} \frac{\partial}{\partial t} \log h(t, y)\right\} .
\end{aligned}
$$

Corollary 2.2. Let $u \in \mathcal{H}(\mathbb{I})$ and $h \in \mathcal{F}(\mathbb{I})$. Then $J(t)=\left\langle u^{2}(t, \cdot), h(t, \cdot)\right\rangle$ is non-increasing on $\mathbb{I}$. that

Next, some useful functions in $\mathcal{F}(\mathbb{I})$ will be given below. Let $\rho(\cdot)$ be any nonnegative function on $\mathcal{V}$ such

$$
|\rho(x)-\rho(y)| \leq d_{\nu}(x, y) \quad \text { for any } x, y \in \mathcal{V} \text { with } x \sim y .
$$

(In practice, one often chooses $\rho(\cdot)=d_{\mathcal{\nu}}(o, \cdot) \wedge R$ for some $o \in \mathcal{V}$ and $R \geq 0$.) 
Lemma 2.3. Let $\tau>0$. For each $t \geq 0$ and $z \in \mathcal{V}$, set

$$
h(t, z)=\exp \left\{\left(\rho(z)-4^{-1} e(t+\tau)\right) \log \left(1 \vee \frac{\rho(z)}{4^{-1} e(t+\tau)}\right)-\frac{t}{\tau}\right\} .
$$

Then $h(t, z) \in \mathcal{F}\left(\mathbb{R}_{+}\right)$.

Proof. We first show that for any $x \in[0, \infty)$ and $\varepsilon \in[0,1]$,

$$
e^{\varepsilon x}+e^{-\varepsilon x}-2 \leq \varepsilon^{2}\left(e^{x}+e^{-x}-2\right)
$$

and

$$
1-e^{-\varepsilon x} \geq \varepsilon\left(1-e^{-x}\right) .
$$

By the Mean Value Theorem,

$$
\frac{e^{\varepsilon x}+e^{-\varepsilon x}-2}{\varepsilon^{2}\left(e^{x}+e^{-x}-2\right)}=\frac{e^{\varepsilon x_{1}}-e^{-\varepsilon x_{1}}}{\varepsilon\left(e^{x_{1}}-e^{-x_{1}}\right)}=\frac{e^{\varepsilon x_{2}}+e^{-\varepsilon x_{2}}}{e^{x_{2}}+e^{-x_{2}}} \leq 1,
$$

where $x>x_{1}>x_{2}>0$. Consequently, (2.4) holds. In the same way, we can obtain (2.5).

Fix $y \sim z$ and $\varepsilon=d_{v}(y, z)$. Then $|\rho(y)-\rho(z)| \leq \varepsilon \leq 1$ by (1.2) and (2.3). Write $t^{+}=t+\tau$ and

$$
b=\left|\left(\rho(y)-4^{-1} e t^{+}\right) \log \left(1 \vee \frac{\rho(y)}{4^{-1} e t^{+}}\right)-\left(\rho(z)-4^{-1} e t^{+}\right) \log \left(1 \vee \frac{\rho(z)}{4^{-1} e t^{+}}\right)\right| .
$$

Then

$$
\frac{|h(t, z)-h(t, y)|^{2}}{4 h(t, z) h(t, y)}=\frac{e^{b}+e^{-b}-2}{4} .
$$

We shall consider three cases.

Case I: $\rho(z), \rho(y) \leq 4^{-1} e t^{+}$. Then $b=0$ and

$$
\frac{|h(t, z)-h(t, y)|^{2}}{4 h(t, z) h(t, y)}=\frac{e^{b}+e^{-b}-2}{4}=0
$$

Case II: $\rho(z), \rho(y) \geq 4^{-1} e t^{+}$. By the Mean Value Theorem,

$$
b=|\rho(y)-\rho(z)|\left(\log \left(\frac{\xi}{4^{-1} e t^{+}}\right)+\frac{\xi-4^{-1} e t^{+}}{\xi}\right),
$$

where $\xi$ is some value between $\rho(y)$ and $\rho(z)$. Furthermore, we have $4^{-1} e t^{+} \leq \xi \leq \rho(y)+\varepsilon$ and

$$
\begin{aligned}
b & \leq \varepsilon \log \left(\frac{4 \xi}{t^{+}} e^{-4^{-1} e t^{+} / \xi}\right) \\
& \leq \varepsilon \log \left(\frac{4 \xi}{t^{+}}\left(1-\left(1-e^{-1}\right) 4^{-1} e t^{+} / \xi\right)\right) \text { by }(2.5) \\
& =\varepsilon \log \left(\frac{4 \xi}{t^{+}}-e+1\right) \leq \varepsilon \log \left(4 \frac{\rho(y)+\varepsilon}{t^{+}}-e+1\right) .
\end{aligned}
$$

As a result,

$$
e^{b}+e^{-b}-2 \leq \exp \left(\varepsilon \log \left(4 \frac{\rho(y)+\varepsilon}{t^{+}}-e+1\right)\right)+\exp \left(-\varepsilon \log \left(4 \frac{\rho(y)+\varepsilon}{t^{+}}-e+1\right)\right)-2 .
$$


Using (2.4) we get

$$
\begin{aligned}
e^{b}+e^{-b}-2 & \leq \varepsilon^{2}\left\{\left(4 \frac{\rho(y)+\varepsilon}{t^{+}}-e+1\right)+\left(4 \frac{\rho(y)+\varepsilon}{t^{+}}-e+1\right)^{-1}-2\right\} \\
& \leq \varepsilon^{2}\left\{4 \frac{\rho(y)+\varepsilon}{t^{+}}-e\right\},
\end{aligned}
$$

and hence

$$
\frac{|h(t, z)-h(t, y)|^{2}}{4 h(t, z) h(t, y)} \leq \varepsilon^{2}\left(\frac{\rho(y)}{t^{+}}+\frac{\varepsilon}{t^{+}}-\frac{e}{4}\right) \leq \varepsilon^{2}\left(\frac{\rho(y)}{t^{+}}+\frac{1}{\tau}-\frac{e}{4}\right) .
$$

Case III: $\rho(y) \wedge \rho(z)<4^{-1} e t^{+}<\rho(y) \vee \rho(z)$. Since $|\rho(y)-\rho(z)| \leq \varepsilon$, we have

$$
\rho(y)+\varepsilon \geq \rho(y) \vee \rho(z)>4^{-1} e t^{+} \quad \text { and } \quad \rho(y) \vee \rho(z)-4^{-1} e t^{+}<\varepsilon .
$$

It implies

$$
4 \frac{\rho(y)+\varepsilon}{t^{+}}-\frac{\rho(y)+\varepsilon}{4^{-1} e t^{+}}=\frac{\rho(y)+\varepsilon}{4^{-1} e t^{+}}(e-1) \geq e-1 .
$$

Hence

$$
\begin{aligned}
b & =\left|\left(\rho(z) \vee \rho(y)-4^{-1} e t^{+}\right) \log \left(\frac{\rho(z) \vee \rho(y)}{4^{-1} e t^{+}}\right)\right| \\
& \leq \varepsilon \log \left(\frac{\rho(y)+\varepsilon}{4^{-1} e t^{+}}\right) \leq \varepsilon \log \left(4 \frac{\rho(y)+\varepsilon}{t^{+}}-e+1\right) .
\end{aligned}
$$

Similarly, we have (2.6) for this case.

On the other hand, note that $h(\cdot, y)$ is differentiable on $\mathbb{R}^{+}$and satisfies

$$
\begin{aligned}
-\frac{\partial}{\partial t} \log h(t, y) & =-\frac{\partial}{\partial t}\left(\left(\rho(y)-4^{-1} e t^{+}\right) \log \left(1 \vee \frac{\rho(y)}{4^{-1} e t^{+}}\right)-\frac{t}{\tau}\right) \\
& =\frac{1}{\tau}+4^{-1} e \log \left(1 \vee \frac{\rho(y)}{4^{-1} e t^{+}}\right)+\frac{\left(\rho(y)-4^{-1} e t^{+}\right) \vee 0}{t^{+}} \\
& \geq \frac{1}{\tau}+\left(\frac{\rho(y)}{t^{+}}-\frac{e}{4}\right) \vee 0 .
\end{aligned}
$$

Therefore, in any case we have

$$
\frac{|h(t, z)-h(t, y)|^{2}}{4 h(t, z) h(t, y)} \leq \varepsilon^{2}\left(\frac{1}{\tau}+\left(\frac{\rho(y)}{t^{+}}-\frac{e}{4}\right) \vee 0\right) \leq-\varepsilon^{2} \frac{\partial}{\partial t} \log h(t, y),
$$

which implies $h \in \mathcal{F}\left(\mathbb{R}_{+}\right)$.

The following two examples can be obtained in a similar way as Lemma 2.3 and we leave it to the reader. See the examples in [8, Proposition 2.5 and Theorem 4.1] for a reference.

Example 2.4. Fix $a \in\left[0, \frac{1}{4}\right]$. Let $h_{1}(t, x)=e^{a \rho(x)-\left(a^{2} / 2\right) t}$. Then $h_{1} \in \mathcal{F}\left(\mathbb{R}_{+}\right)$.

Example 2.5. Fix $D \geq 5, R \geq 1, \Delta \geq \frac{24 R}{D}$ and $s>0$. For each $t \in[0, s]$ and $x \in \mathcal{V}$, set $h_{2}(t, x)=\exp \left(-\frac{\rho(x)^{2}}{D(s-t+\Delta)}\right)$. If $1 \leq \rho(x) \leq R$ for each $x \in \mathcal{V}$, then $h_{2} \in \mathcal{F}([0, s])$. 
Now, fix $o \in \mathcal{V}$ and for each $R \geq 0$ set

$$
\mathcal{G}_{R}(\mathbb{I})=\left\{g: g \text { is a function on } \mathbb{I} \times \mathbb{R}_{+}, g(t, r) \text { is non-decreasing in } r, g\left(\cdot, d_{v}(o, \cdot) \wedge R\right) \in \mathcal{F}(\mathbb{I})\right\} .
$$

For brevity, we write $B_{R}=\left\{z \in \mathcal{V}: d_{v}(o, z)<R\right\}$. The lemma below shows the way we use Corollary 2.2.

Lemma 2.6. Let $T \geq \tau \geq 0$ and $R \geq r \geq 0$. Let $u \in \mathcal{H}([\tau, T])$ and $g \in \mathcal{G}_{R}([\tau, T])$. Then

$$
\left\langle u(T, \cdot)^{2}, 1-1_{B_{R}}\right\rangle \leq \frac{g(\tau, r)}{g(T, R)}\|u(\tau, \cdot)\|^{2}+\frac{g(\tau, R)}{g(T, R)}\left\langle u(\tau, \cdot)^{2}, 1-1_{B_{r}}\right\rangle .
$$

Proof. Let $\rho(z)=\min \left\{d_{v}(o, z), R\right\}$ for each $z \in \mathcal{V}$. Then $\rho=R$ on $\mathcal{V} \backslash B_{R}$ and hence

$$
\left\langle u(T, \cdot)^{2}, 1-1_{B_{R}}\right\rangle \leq\left\langle u(T, \cdot)^{2}, g(T, \rho(\cdot))\right| g(T, R)^{-1} .
$$

By Corollary 2.2 and the hypothesis $u \in \mathcal{H}([\tau, T])$ and $g(\cdot, \rho(\cdot)) \in \mathcal{F}([\tau, T])$, we have

$$
\left\langle u(T, \cdot)^{2}, g(T, \rho)\right\rangle \leq\left\langle u(\tau, \cdot)^{2}, g(\tau, \rho)\right\rangle .
$$

Using the condition that $g(t, \cdot)$ is a non-decreasing function, we get

$$
\begin{aligned}
\left\langle u(\tau, \cdot)^{2}, g(\tau, \rho)\right\rangle & \leq\left\langle u(\tau, \cdot)^{2}, 1_{B_{r}}\right\rangle g(\tau, r)+\left\langle u(\tau, \cdot)^{2}, 1-1_{B_{r}}\right\rangle g(\tau, R) \\
& \leq g(\tau, r)\|u(\tau, \cdot)\|^{2}+g(\tau, R)\left\langle u(\tau, \cdot)^{2}, 1-1_{B_{r}}\right\rangle,
\end{aligned}
$$

proving the lemma.

Furthermore, we set

$$
\mathcal{H}_{o}=\left\{u \in \mathcal{H}\left(\mathbb{R}_{+}\right): u(0, z)=v_{o}^{-1 / 2} 1_{\{o\}}(z) \text { for each } z \in \mathcal{V}\right\} .
$$

Proposition 2.7. Let $u \in \mathcal{H}_{o}$. For any $t, R>0$, we have

$$
\left\langle u(t, \cdot)^{2}, 1-1_{B_{R}}\right\rangle \leq \begin{cases}\exp \left(-\frac{R^{2}}{8 t}\right) & \text { if } t \geq R, \\ \exp \left(-R \log \left(\frac{1.01 R}{t}\right)+120\right) & \text { if } t \leq R .\end{cases}
$$

Proof. Consider $t \geq R$ first. Take $a=\frac{R}{4 t}$ then $a \in\left(0, \frac{1}{4}\right]$. For each $s \geq 0$ and $r \geq 0$, set

$$
g_{1}(s, r)=e^{a r-\left(a^{2} / 2\right) s} .
$$

By Example 2.4, $g_{1} \in \mathcal{G}_{R}\left(\mathbb{R}_{+}\right)$. Use Lemma 2.6 and get for $r \in(0, R]$,

$$
\left\langle u(t, \cdot)^{2}, 1-1_{B_{R}}\right\rangle \leq \frac{g_{1}(0, r)}{g_{1}(t, R)}\|u(0, \cdot)\|^{2}+\frac{g_{1}(0, R)}{g_{1}(t, R)}\left\langle u(0, \cdot)^{2}, 1-1_{B_{r}}\right\rangle .
$$

From $u(0, z)=v_{o}^{-1 / 2} 1_{\{o\}}(z)$, it follows immediately that

$$
\left\langle u(0, \cdot)^{2}, 1-1_{B_{r}}\right\rangle=0 \quad \text { and } \quad\|u(0, \cdot)\|^{2}=1 .
$$

So,

$$
\left\langle u(t, \cdot)^{2}, 1-1_{B_{R}}\right\rangle \leq \lim _{r \rightarrow 0+} \frac{g_{1}(0, r)}{g_{1}(t, R)}=\frac{g_{1}(0,0)}{g_{1}(t, R)} .
$$


Obviously, $g_{1}(0,0)=1$ and hence

$$
\left\langle u^{2}(t, \cdot), 1-1_{B_{R}}\right\rangle \leq e^{-a R+\left(a^{2} / 2\right) t} .
$$

Substituting the value of $a$ into the above, we get the first inequality of the proposition.

Next, suppose $t \leq R$. Choose $\tau=(4 c / e-1) t$, where $b=(4 c / e-1)^{-1} \approx 117.6$ and $c=e^{-e^{-1}} / 1.01$. For each $s \geq 0$ and $r \geq 0$, set

$$
g_{2}(s, r)=\exp \left\{\left(r-4^{-1} e(s+\tau)\right) \log \left(1 \vee \frac{r}{4^{-1} e(s+\tau)}\right)-\frac{s}{\tau}\right\} .
$$

Obviously, $g_{2}(0,0)=1$. By Lemma 2.3, we have $g_{2} \in \mathcal{G}_{R}\left(\mathbb{R}_{+}\right)$. Since $x \log (R / x) \leq e^{-1} R$ for any $x>0$, we get

$$
\begin{aligned}
\log \left(g_{2}(t, R)\right) & =(R-c t) \log \left(\frac{R}{c t}\right)-b \\
& =R \log \left(\frac{1.01 R}{t}\right)+R \log \left(\frac{1}{1.01 c}\right)-c t \log \left(\frac{R}{c t}\right)-b \\
& \geq R \log \left(\frac{1.01 R}{t}\right)+R \log \left(\frac{1}{1.01 c}\right)-e^{-1} R-120 \\
& =R \log \left(\frac{1.01 R}{t}\right)-120 .
\end{aligned}
$$

From (2.7) and $g_{2} \in \mathcal{G}_{R}\left(\mathbb{R}_{+}\right)$, we prove the second inequality of the proposition in the same way as we did the first.

Corollary 2.8. For any $z \in \mathcal{V}$,

$$
\mathbb{P}_{o}\left(X_{t}=z\right) \leq \begin{cases}\left(v_{z} / v_{o}\right)^{1 / 2} \exp \left\{-\frac{r^{2}}{16 t}\right\} & \text { if } t \geq r>0, \\ \left(v_{z} / v_{o}\right)^{1 / 2} \exp \left(-\frac{r}{2} \log \left(\frac{1.01 r}{t}\right)+60\right) & \text { if } r \geq t>0,\end{cases}
$$

where $r=d_{v}(o, z)$.

Proof. Recall the definition $u_{B}$ in (2.1). Denote by $d(\cdot, \cdot)$ the graph distance of $\Gamma$. Set $S_{n}=\{z: d(o, z)<n\}$. Then $S_{n}$ is a finite set since $\Gamma$ is a locally finite graph and hence $u_{S_{n}} \in \mathcal{H}_{o}$. Clearly, $u_{S_{n}}$ converges pointwise to $u$ as $n$ tends to infinity even if the process $X$ explodes in finite time, where

$$
u(t, z)=\frac{v_{o}^{1 / 2}}{v_{z}} \mathbb{P}_{o}\left(X_{t}=z\right) .
$$

Let $r=d_{v}(o, z)$, then we have $\left\langle u_{S_{n}}(t, \cdot)^{2}, 1-1_{B_{r}}\right\rangle \geq u_{S_{n}}(t, z)^{2} v_{z}$. So,

$$
u(t, z)^{2} v_{z}=\lim _{n \rightarrow \infty} u_{S_{n}}(t, z)^{2} v_{z} \leq \sup _{n}\left\langle u_{S_{n}}(t, \cdot)^{2}, 1-1_{B_{r}}\right\rangle .
$$

Combining the above inequality with Proposition 2.7, we get the desired result.

The long range bounds for transition probabilities were already obtained by [11, Theorems 2.1 and 2.2], however, Corollary 2.8 is more effective when $t \in[0.9 r, 1.1 r]$ and $r=d_{\nu}(o, z)$ is large.

\section{Regular functions and integral estimates}

Recall that $A \geq 1$ and $\gamma>1$. Fix $\delta \geq 1, \theta_{1}=10^{-6}$ and $\theta_{2}=\theta_{1} / 5$. Set

$$
\alpha=\min \left\{(2 \gamma)^{-1},(64 \delta)^{-1}\right\} \quad \text { and } \quad \beta=\left\lceil\frac{\log 2}{\log \gamma}\right\rceil .
$$


Let $u \in \mathcal{H}_{o}$ and $f: \mathbb{R}_{+} \mapsto \mathbb{R}_{+}$such that

$$
\|u(t, \cdot)\|^{2} \leq \frac{1}{f(2 t)} \quad \text { for all } t \in \mathbb{R}_{+} .
$$

In this section, we shall extend Proposition 2.7 into a result which can be used to prove Theorem 1.1.

Proposition 3.1. Suppose $f$ is $(A, \gamma)$-regular and satisfies $f(t) \leq A e^{\delta t}$ for all $t \in \mathbb{R}_{+}$. Then there exists a constant $C_{1}>0$ which is independent of $A, \gamma$ and $\delta$, such that for $t>0$,

$$
\left\langle u(t, \cdot)^{2}, \exp \left(\theta_{2} \frac{\left(d_{v}(o, \cdot) \wedge(2 t)\right)^{2}}{t}\right)\right\rangle \leq \frac{C_{1} A^{\beta}}{f(2 \alpha t)} .
$$

Before proving the proposition, we establish some lemmas.

Lemma 3.2. If $f$ is an $(A, \gamma)$-regular function, then

$$
f\left(2^{-k} t\right) \geq\left(A^{\beta} \frac{f(t)}{f\left(\gamma^{-\beta} t\right)}\right)^{-k} f(t) \quad \text { for all } k \in \mathbb{N} \text { and } t>0 .
$$

Proof. By the regularity, for any $t \geq s>0$ we have

$$
\frac{f\left(\gamma^{\beta} s\right)}{f(s)}=\prod_{j=0}^{\beta-1} \frac{f\left(\gamma^{j+1} s\right)}{f\left(\gamma^{j} s\right)} \leq \prod_{j=0}^{\beta-1}\left(A \frac{f\left(\gamma^{j+1} t\right)}{f\left(\gamma^{j} t\right)}\right)=A^{\beta} \frac{f\left(\gamma^{\beta} t\right)}{f(t)} .
$$

In other words, an $(A, \gamma)$-regular function is also $\left(A^{\beta}, \gamma^{\beta}\right)$-regular. Furthermore, by the monotonicity we get

$$
\frac{f(t)}{f\left(2^{-k} t\right)} \leq \frac{f(t)}{f\left(\gamma^{-\beta k} t\right)}=\prod_{j=-k}^{-1} \frac{f\left(\gamma^{\beta(j+1)} t\right)}{f\left(\gamma^{\beta j} t\right)} \leq \prod_{j=-k}^{-1}\left(A^{\beta} \frac{f(t)}{f\left(\gamma^{-\beta} t\right)}\right)=\left(A^{\beta} \frac{f(t)}{f\left(\gamma^{-\beta} t\right)}\right)^{k} .
$$

Lemma 3.3. If $f(t) \leq A e^{\delta t}$ for each $t \in \mathbb{R}_{+}$, then there exists a constant $c>0$ which is independent of $A$ and $\delta$, such that

$$
\left\langle u(t, \cdot)^{2}, 1-1_{B_{R}}\right\rangle \leq \frac{c A}{f(R /(32 \delta))} e^{-10^{-4} R} \text { for } t>0 \text { and } R \in[t, 64 t]
$$

Proof. Fix $t>0, R \in[t, 64 t], x=t / R$ and $a=(64 \delta)^{-1}$. Then $a \leq x \leq 1$. Write $a_{1}=4^{-1} e(a+0.45)$ and $b=$ $4^{-1} e(x+0.45)$. Then,

$$
a_{1} \geq 4^{-1} e \cdot 0.45 \geq 0.3 \quad \text { and } \quad a_{1} \leq b \leq 4^{-1} e(1+0.45) \leq 0.99
$$

For each $s \geq 0$ and $r \geq 0$, we define

$$
g(s, r)=\exp \left\{\left(r-4^{-1} e(s+0.45 R)\right) \log \left(1 \vee \frac{r}{4^{-1} e(s+0.45 R)}\right)-\frac{s}{0.45 R}\right\} .
$$

By Lemma 2.3, we have $g \in \mathcal{G}_{R}\left(\mathbb{R}_{+}\right)$. Applying Lemma 2.6 gives

$$
\left\langle u(t, \cdot)^{2}, 1-1_{B_{R}}\right\rangle \leq \frac{g\left(a R, a_{1} R\right)}{g(x R, R)}\|u(a R, \cdot)\|^{2}+\frac{g(a R, R)}{g(x R, R)}\left\langle u(a R, \cdot)^{2}, 1-1_{B_{a_{1} R}}\right\rangle .
$$

By a direct calculation, we get $g\left(a R, a_{1} R\right) \leq 1$,

$$
\log (g(a R, R)) \leq R\left(1-a_{1}\right) \log \left(1 \vee \frac{1}{a_{1}}\right) \leq R(1-0.3) \log \left(\frac{1}{0.3}\right) \leq 0.8428 R,
$$


and

$$
\log (g(x R, R))=R(1-b) \log \left(\frac{1}{b}\right)-\frac{x}{0.45} \geq R(1-0.99) \log \left(\frac{1}{0.99}\right)-3 \geq 0.0001 R-3 .
$$

Thus, (3.4) becomes

$$
\left\langle u(t, \cdot)^{2}, 1-1_{B_{R}}\right\rangle \leq e^{-0.0001 R+3}\|u(a R, \cdot)\|^{2}+e^{0.843 R+3}\left\langle u(a R, \cdot)^{2}, 1-1_{B_{a_{1} R}}\right\rangle .
$$

By (3.1) and the hypothesis $f(s) \leq A e^{\delta s}$, we obtain,

$$
\left\langle u(t, \cdot)^{2}, 1-1_{B_{R}}\right\rangle \leq \frac{1}{f(2 a R)} e^{-0.0001 R+3}+\frac{A e^{2 a \delta R}}{f(2 a R)} e^{0.843 R+3}\left\langle u(a R, \cdot)^{2}, 1-1_{B_{a_{1} R}}\right\rangle .
$$

By Proposition 2.7,

$$
\left\langle u(a R, \cdot)^{2}, 1-1_{B_{a_{1} R}}\right\rangle \leq \exp \left(-a_{1} R \log \left(\frac{a_{1}}{a}\right)+120\right) .
$$

Therefore,

$$
\left\langle u(t, \cdot)^{2}, 1-1_{B_{R}}\right\rangle \leq \frac{A e^{123}}{f(2 a R)}\left(e^{-0.0001 R}+\exp \left(2 a \delta R+0.843 R-a_{1} R \log \left(\frac{a_{1}}{a}\right)\right)\right) .
$$

Substitute $a=(64 \delta)^{-1}$ and get,

$$
\left\langle u(t, \cdot)^{2}, 1-1_{B_{R}}\right\rangle \leq \frac{A e^{123}}{f(R /(32 \delta))}\left(e^{-0.0001 R}+e^{-R C}\right),
$$

where $C=a_{1} \log \left(64 a_{1} \delta\right)-0.8743$. Since $a_{1} \geq 0.3$ and $\delta \geq 1$, we have $C \geq 0.01$. So,

$$
\left\langle u(t, \cdot)^{2}, 1-1_{B_{R}}\right\rangle \leq \frac{2 A e^{123}}{f(R /(32 \delta))} e^{-0.0001 R} .
$$

Proposition 3.4. Suppose that $f$ is $(A, \gamma)$-regular and satisfies $f(t) \leq A e^{\delta t}$ for all $t \in \mathbb{R}_{+}$. Then there exists a constant $C_{0}>0$ which is independent of $A, \gamma$ and $\delta$, such that

$$
\left\langle u(t, \cdot)^{2}, 1-1_{B_{R}}\right\rangle \leq \frac{C_{0} A^{\beta}}{f(2 \alpha t)} \exp \left(-\theta_{1} \frac{R^{2}}{t}\right) \quad \text { for all } t \geq R \geq 10^{3} .
$$

Proof. Fix $L=\log \left(A^{\beta} \frac{f(2 t)}{f\left(2 t / \gamma^{\beta}\right)}\right), D=100$ and $\Delta=\frac{R}{4}$. If $\theta_{1} \frac{R^{2}}{t}-L-\frac{1}{D \Delta}<\theta_{1}$, then we complete the proof since

$$
\begin{aligned}
\left\langle u(t, \cdot)^{2}, 1-1_{B_{R}}\right\rangle & \leq\|u(t, \cdot)\|^{2} \\
& \leq \frac{1}{f(2 t)} \leq \frac{e^{\theta_{1}}}{f(2 t)} \exp \left(L+\frac{1}{D \Delta}-\theta_{1} \frac{R^{2}}{t}\right) \\
& =\frac{e^{\theta_{1}} A^{\beta} \exp (1 /(D \Delta))}{f\left(2 t / \gamma^{\beta}\right)} \exp \left(-\theta_{1} \frac{R^{2}}{t}\right) \\
& \leq \frac{e^{\theta_{1}} A^{\beta} \exp (1 / 100)}{f(t / \gamma)} \exp \left(-\theta_{1} \frac{R^{2}}{t}\right),
\end{aligned}
$$

where the last inequality uses the monotonicity of $f$. Therefore, we may assume that

$$
t \geq R \geq 10^{3} \quad \text { and } \quad \theta_{1} \frac{R^{2}}{t}-L-\frac{1}{D \Delta} \geq \theta_{1} .
$$


This implies that $R \leq t \leq R^{2}$ and $L \leq \theta_{1} \frac{R^{2}}{t}$.

Let $\rho(x)=\left(R-d_{v}(o, x)\right) \vee 1$ for any $x \in \mathcal{V}$. Then $\rho$ satisfies (2.3) and $1 \leq \rho(x) \leq R$. For each $s \in[0, t]$ and $r \geq 0$, set

$$
g(s, r)=\exp \left(-\frac{((R-r) \vee 1)^{2}}{D(t-s+\Delta)}\right) .
$$

Then $g \in \mathcal{G}_{R}([0, t])$ by Example 2.5 and the argument above about $\rho$. From Lemma 2.6, we get that for any $r \in[0, R]$ and $s \in[0, t]$,

$$
\begin{aligned}
\left\langle u(t, \cdot)^{2}, 1-1_{B_{R}}\right\rangle & \leq \frac{g(s, r)}{g(t, R)}\|u(s, \cdot)\|^{2}+\frac{g(s, R)}{g(t, R)}\left\langle u(s, \cdot)^{2}, 1-1_{B_{r}}\right\rangle \\
& \leq \frac{\exp (1 /(D \Delta))}{f(2 s)} \exp \left(-\frac{(R-r)^{2}}{D(t-s+\Delta)}\right)+\exp \left(\frac{1}{D \Delta}\right)\left\langle u(s, \cdot)^{2}, 1-1_{B_{r}}\right\rangle .
\end{aligned}
$$

We shall iterate using (3.6). Let us build a sequence $\left\{\left(t_{j}, R_{j}\right): 0 \leq j \leq j_{0}\right\}$. Take

$$
t_{j}=t / 2^{j-1}, \quad R_{j}=R / 2+R /(j+1) \quad \text { for each } 0 \leq j \leq j_{0} ;
$$

and

$$
j_{0}=\min \left\{j: R_{j} \geq t_{j}\right\} .
$$

Then $j_{0} \geq 1$ and for all $0 \leq j<j_{0}$ we have $t_{j}>R_{j}>R / 2>1$. Hence

$$
t_{j}-t_{j+1}=t_{j} / 2 \geq R / 4=\Delta .
$$

From $t_{j_{0}-1}>R / 2$, we get

$$
j_{0}<\frac{\log (8 t / R)}{\log 2} \text {. }
$$

Using the identity $\left(R_{j}-R_{j+1}\right)^{2}=\frac{R^{2}}{(j+1)^{2}(j+2)^{2}}$, we obtain

$$
\frac{\left(R_{j}-R_{j+1}\right)^{2}}{D\left(t_{j}-t_{j+1}+\Delta\right)} \geq \frac{\left(R_{j}-R_{j+1}\right)^{2}}{D t_{j}}=\frac{2^{j-1}}{D(j+1)^{2}(j+2)^{2}} \frac{R^{2}}{t} .
$$

Note that

$$
\min \left\{\frac{2^{j-1}}{100(j+1)^{3}(j+2)^{2}}: j \geq 1\right\}=\frac{2^{6-1}}{100(6+1)^{3}(6+2)^{2}} \approx 1.5 \times 10^{-5} .
$$

Sicne $\theta_{1}=10^{-6}$, it follows immediately that

$$
\frac{\left(R_{j}-R_{j+1}\right)^{2}}{D\left(t_{j}-t_{j+1}+\Delta\right)} \geq(j+1) \theta_{1} \frac{R^{2}}{t} .
$$

Iterating (3.6), we obtain

$$
\begin{aligned}
\left\langle u(t, \cdot)^{2}, 1-1_{B_{R}}\right\rangle & =\left\langle u\left(t_{1}, \cdot\right)^{2}, 1-1_{B_{R_{1}}}\right\rangle \\
& \leq \sum_{j=1}^{j_{0}-1} \frac{\exp (j /(D \Delta))}{f\left(2 t_{j+1}\right)} \exp \left(-\frac{\left(R_{j}-R_{j+1}\right)^{2}}{D\left(t_{j}-t_{j+1}+\Delta\right)}\right)+\exp \left(\frac{j_{0}-1}{D \Delta}\right)\left\langle u\left(t_{j_{0}}, \cdot\right)^{2}, 1-1_{B_{R_{j}}}\right\rangle \\
& :=\Lambda_{1}+\Lambda_{2} .
\end{aligned}
$$


By Lemma 3.2, we have

$$
f\left(2 t_{j+1}\right) \geq f(2 t) e^{-j L} .
$$

Using (3.5), we conclude

$$
\begin{aligned}
\Lambda_{1} & \leq \frac{1}{f(2 t)} \exp \left(-\theta_{1} \frac{R^{2}}{t}\right) \sum_{j=1}^{j_{0}-1} \exp \left(-j\left(\theta_{1} \frac{R^{2}}{t}-L-\frac{1}{D \Delta}\right)\right) \\
& \leq \frac{1}{f(2 t)} \exp \left(-\theta_{1} \frac{R^{2}}{t}\right) \sum_{j=1}^{j_{0}-1} \exp \left(-j \theta_{1}\right) \\
& \leq \frac{e^{-\theta_{1}}\left(1-e^{-\theta_{1}}\right)^{-1}}{f(2 t)} \exp \left(-\theta_{1} \frac{R^{2}}{t}\right) .
\end{aligned}
$$

On the other hand, since $2 t_{j_{0}}=t_{j_{0}-1}>R_{j_{0}-1}>R_{j_{0}} \geq t_{j_{0}}$, we use Lemma 3.3 to get

$$
\left\langle u\left(t_{j_{0}}, \cdot\right)^{2}, 1-1_{B_{R_{0}}}\right\rangle \leq \frac{c A}{f\left(R_{j_{0}} /(32 \delta)\right)} e^{-10^{-4} R_{j_{0}}}
$$

where $c$ is a constant which is independent of $A, \gamma$ and $\delta$. By Lemma 3.2 and (3.3), we also have

$$
\begin{aligned}
f\left(\frac{R_{j_{0}}}{32 \delta}\right) & \geq f\left(\frac{t_{j_{0}}}{32 \delta}\right) \geq\left(A^{\beta} \frac{f(t /(32 \delta))}{f\left(t /\left(32 \delta \gamma^{\beta}\right)\right)}\right)^{-j_{0}+1} f\left(\frac{t}{32 \delta}\right) \\
& \geq\left(A^{2 \beta} \frac{f(2 t)}{f\left(2 t / \gamma^{\beta}\right)}\right)^{-j_{0}+1} f\left(\frac{t}{32 \delta}\right) \\
& \geq f\left(\frac{t}{32 \delta}\right) e^{-2 j_{0} L} .
\end{aligned}
$$

So,

$$
\begin{aligned}
\Lambda_{2} & =\exp \left(\frac{j_{0}-1}{D \Delta}\right)\left\langle u\left(t_{j_{0}}, \cdot\right)^{2}, 1-1_{B_{R_{j_{0}}}}\right\rangle \leq \exp \left(\frac{j_{0}-1}{D \Delta}\right) \frac{c A}{f\left(R_{j_{0}} /(32 \delta)\right)} e^{-10^{-4} R_{j_{0}}} \\
& \leq \frac{c A}{f(t /(32 \delta))} \exp \left(\frac{j_{0}}{D \Delta}+2 j_{0} L-10^{-4} R / 2\right) .
\end{aligned}
$$

Note that

$$
10^{3} \leq R \leq t \leq R^{2}, \quad j_{0}<\frac{\log (8 t / R)}{\log 2}, \quad D \Delta=25 R \quad \text { and } \quad L \leq \theta_{1} \frac{R^{2}}{t} .
$$

From these inequalities, we calculate

$$
\begin{aligned}
& \frac{j_{0}}{D \Delta R} \leq \frac{\log (8 t / R)}{25 R^{2} \log 2} \leq \frac{\log (8 R)}{25 R^{2} \log 2} \leq \frac{\log \left(8 \cdot 10^{3}\right)}{25 \cdot 10^{6} \cdot \log 2}<5.2 \times 10^{-7} \\
& \frac{2 j_{0} L}{R} \leq 2 \theta_{1} \frac{\log (8 t / R)}{\log 2} \frac{R}{t} \leq 2 \theta_{1} \frac{8}{e \log 2}<8.5 \times 10^{-6} .
\end{aligned}
$$

So, $\frac{j_{0}}{D \Delta}+2 j_{0} L-10^{-4} R / 2<-\theta_{1} R$ and hence

$$
\Lambda_{2} \leq \frac{c A}{f(t /(32 \delta))} e^{-\theta_{1} R} \leq \frac{c A}{f(t /(32 \delta))} e^{-\theta_{1} R^{2} / t} .
$$


Finally, we choose

$$
C_{0}=e^{\theta_{1}+0.01}+e^{-\theta_{1}}\left(1-e^{-\theta_{1}}\right)^{-1}+c
$$

and complete the proof.

Proof of Proposition 3.1. Write $\rho(z)=d_{v}(o, z) \wedge(2 t)$ for short. If $t \leq 10^{6}$, then the result is trivial since

$$
\left\langle u(t, \cdot)^{2}, \exp \left(\theta_{2} \frac{\rho^{2}}{t}\right)\right\rangle \leq e^{4 \theta_{2} t}\|u(t, \cdot)\|^{2} \leq \frac{e^{4 \cdot 10^{6} \theta_{2}}}{f(2 t)} .
$$

So, we may assume that $t \geq 10^{6}$ in the following.

Fix $R=t^{1 / 2}$ and $n=\left\lceil\frac{\log (t / R)}{\log 2}\right\rceil$. Then $2^{n} R \geq t$, and $t \geq 2^{j-1} R \geq 10^{3}$ for each $1 \leq j \leq n$. Write

$$
\Upsilon_{0}=\left\langle u(t, \cdot)^{2}, e^{\theta_{2} \rho^{2} / t} 1_{B_{R}}\right\rangle, \quad \Upsilon_{\infty}=\left\langle u(t, \cdot)^{2}, e^{\theta_{2} \rho^{2} / t}\left(1-1_{B_{t}}\right)\right\rangle
$$

and set

$$
\Upsilon_{j}=\left\langle u(t, \cdot)^{2}, e^{\theta_{2} \rho^{2} / t}\left(1_{B_{2^{j} R}}-1_{B_{2^{j-1} R}}\right)\right\rangle \quad \text { for } 1 \leq j \leq n .
$$

Then

$$
\left\langle u(t, \cdot)^{2}, \exp \left(\theta_{2} \frac{\rho^{2}}{t}\right)\right\rangle \leq \Upsilon_{0}+\sum_{j=1}^{n} \Upsilon_{j}+\Upsilon_{\infty} .
$$

We estimate each $\Upsilon_{j}$ separately.

The first term admits the estimate

$$
\Upsilon_{0} \leq\left\langle u(t, \cdot)^{2}, e^{\theta_{2}} 1_{B_{R}}\right\rangle \leq e^{\theta_{2}}\|u(t, \cdot)\|^{2} \leq \frac{e^{\theta_{2}}}{f(2 t)} .
$$

Next, for each $1 \leq j \leq n$, we have

$$
\Upsilon_{j} \leq\left\langle u(t, \cdot)^{2}, e^{\theta_{2}\left(2^{j}\right)^{2}}\left(1_{B_{2 j_{R}}}-1_{B_{2^{j-1} R}}\right)\right\rangle \leq e^{4^{j} \theta_{2}}\left\langle u(t, \cdot)^{2}, 1-1_{B_{2^{j-1} 1_{R}}}\right\rangle .
$$

Set $C_{0}$ as in Proposition 3.4. Then

$$
\left\langle u(t, \cdot)^{2}, 1-1_{B_{2^{j-1}}}\right\rangle \leq \frac{C_{0} A^{\beta}}{f(2 \alpha t)} \exp \left(-\theta_{1} \cdot 4^{j-1}\right) .
$$

By definition $\theta_{2}=\theta_{1} / 5$; therefore we get

$$
\Upsilon_{j} \leq \frac{C_{0} A^{\beta}}{f(2 \alpha t)} \exp \left(-\theta_{2} \cdot 4^{j-1}\right)
$$

For the remaining term,

$$
\Upsilon_{\infty} \leq e^{4 \theta_{2} t}\left\langle u(t, \cdot)^{2},\left(1-1_{B_{t}}\right)\right\rangle .
$$

Using Proposition 3.4 again gives

$$
\Upsilon_{\infty} \leq e^{4 \theta_{2} t} \cdot \frac{C_{0} A^{\beta}}{f(2 \alpha t)} e^{-\theta_{1} t}=\frac{C_{0} A^{\beta}}{f(2 \alpha t)} e^{-\theta_{2} t} \leq \frac{C_{0} A^{\beta}}{f(2 \alpha t)} .
$$


Therefore,

$$
\begin{aligned}
\left\langle u(t, \cdot)^{2}, \exp \left(\theta_{2} \frac{\rho^{2}}{t}\right)\right\rangle & \leq \frac{e^{\theta_{2}}}{f(2 t)}+\sum_{j=1}^{n} \frac{C_{0} A^{\beta}}{f(2 \alpha t)} \exp \left(-\theta_{2} \cdot 4^{j-1}\right)+\frac{C_{0} A^{\beta}}{f(2 \alpha t)} \\
& \leq \frac{C_{1} A^{\beta}}{f(2 \alpha t)},
\end{aligned}
$$

where

$$
C_{1}=e^{4 \cdot 10^{6} \theta_{2}}+C_{0} \sum_{j=1}^{\infty} \exp \left(-\theta_{2} \cdot 4^{j-1}\right)+C_{0} .
$$

\section{Proof of Theorem 1.1}

Proof of Theorem 1.1. Recall the notation $\mathcal{H}$. from Section 2. Fix $t \geq d_{v}\left(x_{1}, x_{2}\right)$ and $s=t / 2$. For each $z \in \mathcal{V}$ and $i \in\{1,2\}$, set

$$
\rho_{i}(s, z)=d_{v}\left(x_{i}, z\right) \wedge(2 s) \quad \text { and } \quad h_{i}(s, z)=\exp \left(\frac{1}{2} \cdot \theta_{2} \frac{\rho_{i}(s, z)^{2}}{s}\right) .
$$

Then $2 \rho_{1}(s, z)^{2}+2 \rho_{2}(s, z)^{2} \geq d_{v}\left(x_{1}, x_{2}\right)^{2}$ and so

$$
h_{1}(s, z) h_{2}(s, z) \geq \exp \left(\frac{\theta_{2}}{2} \cdot \frac{d_{v}\left(x_{1}, x_{2}\right)^{2}}{t}\right) .
$$

Let $d(\cdot, \cdot)$ be the graph distance of $\Gamma$. As in Corollary 2.8, we define

$$
u_{i j}(s, z)=\frac{v_{x_{i}}^{1 / 2}}{v_{z}} \mathbb{P}_{x_{i}}\left(X_{s}=z, \inf \left\{l \in \mathbb{R}_{+}: d\left(x_{i}, X_{l}\right) \geq j\right\}>s\right)
$$

and $u_{i}(s, z)=\frac{v_{x_{i}}^{1 / 2}}{v_{z}} \mathbb{P}_{x_{i}}\left(X_{s}=z\right)$. Then $\left\{u_{i j}(s, z): j=1,2, \ldots\right\}$ is a non-decreasing sequence and satisfies

$$
\lim _{j \rightarrow \infty} u_{i j}(s, z)=u_{i}(s, z) .
$$

By (1.1), for any $l \geq 0$ we have

$$
\left\|u_{i j}(l, \cdot)\right\|^{2} \leq\left\|u_{i}(l, \cdot)\right\|^{2}=\mathbb{P}_{x_{i}}\left(X_{2 l}=x_{i}\right) \leq \frac{1}{f_{i}(2 l)} .
$$

Since $u_{i j} \in \mathcal{H}_{x_{i}}$, we use Proposition 3.1 and get

$$
\left\|u_{i j}(s, \cdot) h_{i}(s, \cdot)\right\|^{2}=\left\langle u_{i j}(s, \cdot)^{2}, \exp \left(\theta_{2} \frac{\rho_{i}(s, \cdot)^{2}}{s}\right)\right\rangle \leq \frac{C_{1} A^{\beta}}{f_{i}(2 \alpha s)}=\frac{C_{1} A^{\beta}}{f_{i}(\alpha t)}
$$

By the Monotone Convergence Theorem,

$$
\left\|u_{i}(s, \cdot) h_{i}(s, \cdot)\right\|^{2}=\lim _{j \rightarrow \infty}\left\|u_{i j}(s, \cdot) h_{i}(s, \cdot)\right\|^{2} \leq \frac{C_{1} A^{\beta}}{f_{i}(\alpha t)} .
$$


By (4.1) and the Cauchy-Schwarz inequality, we obtain

$$
\begin{aligned}
\mathbb{P}_{x_{1}}\left(X_{t}=x_{2}\right) & =\sum_{z \in \mathcal{V}} \mathbb{P}_{x_{1}}\left(X_{s}=z\right) \mathbb{P}_{x_{2}}\left(X_{s}=z\right) \frac{v_{x_{2}}}{v_{z}} \\
& =\left(v_{x_{2}} / v_{x_{1}}\right)^{1 / 2}\left\langle u_{1}(s, \cdot), u_{2}(s, \cdot)\right\rangle \\
& \leq\left(v_{x_{2}} / v_{x_{1}}\right)^{1 / 2}\left\langle u_{1}(s, \cdot) h_{1}(s, \cdot), u_{2}(s, \cdot) h_{2}(s, \cdot)\right\rangle \exp \left(-\frac{\theta_{2}}{2} \cdot \frac{d_{v}\left(x_{1}, x_{2}\right)^{2}}{t}\right) \\
& \leq\left(v_{x_{2}} / v_{x_{1}}\right)^{1 / 2}\left\|u_{1}(s, \cdot) h_{1}(s, \cdot)\right\|\left\|u_{2}(s, \cdot) h_{2}(s, \cdot)\right\| \exp \left(-\frac{\theta_{2}}{2} \cdot \frac{d_{v}\left(x_{1}, x_{2}\right)^{2}}{t}\right) \\
& \leq \frac{C_{1} A^{\beta}\left(v_{x_{2}} / v_{x_{1}}\right)^{1 / 2}}{\sqrt{f_{1}(\alpha t) f_{2}(\alpha t)}} \exp \left(-\frac{\theta_{2}}{2} \cdot \frac{d_{v}\left(x_{1}, x_{2}\right)^{2}}{t}\right) .
\end{aligned}
$$

Set $\theta=\theta_{2} / 2$ and we complete the proof.

\section{Regularity on an interval}

Proof of Theorem 1.2. First, we show that

$$
\left\langle u(t, \cdot)^{2}, \exp \left(\theta_{2} \frac{\left(d_{v}(o, \cdot) \wedge(2 t)\right)^{2}}{t}\right)\right\rangle \leq \frac{C_{1} A^{\beta}}{f(2 \alpha t)} \quad \text { for } t \in\left[\left(2 \alpha^{-1} T_{1}\right)^{2}, T_{2} / 2\right) .
$$

Take $t \in\left[\left(2 \alpha^{-1} T_{1}\right)^{2}, T_{2} / 2\right), t_{j}=t / 2^{j-1}, R_{j}=R / 2+R /(j+1), j_{0}=\min \left\{j: R_{j} \geq t_{j}\right\}$ and $R=t^{1 / 2}$ as in Propositions 3.1 and 3.4. Then

$$
2 \alpha t_{j_{0}}>\alpha R / 2=\alpha t^{1 / 2} / 2 \geq T_{1} .
$$

Using (1.5) and the regular condition on $\left[T_{1}, T_{2}\right.$ ), we still have the inequalities (3.7), (3.9) and (3.10). Therefore, we can get (5.1) in the same way as we did Proposition 3.1. Furthermore, by (5.1) and the Cauchy-Schwarz inequality, we finish the proof of Theorem 1.2 similar as that of Theorem 1.1.

Heat kernels having either polynomial decay or sub-exponential decay appear in many groups, see Hebisch and Saloff-Coste [15, Theorem 4.1]. More importantly, there are a lot of papers which studied random walks on $\mathbb{Z}^{d}$ with random conductances and showed that

$$
\mathbb{P}_{x}\left(X_{t}=x\right) \leq c v_{x} t^{-d / 2} \text { for all } t \geq t_{x}
$$

under different conditions, such as $[1,2,4-6]$. A general feature of these random walks with random conductances is that one may have (5.2) with either $v_{x} \rightarrow 0$ or $t_{x} \rightarrow \infty$ as $x$ goes to infinity. Thus the condition (1.4) fails for small time $t$ and so we cannot apply Theorem 1.1 directly to give a uniform bound on $\mathbb{P}_{x}\left(X_{t}=\cdot\right)$. This is the motivation for our studying the regularity on an interval.

The lower bound of $\widetilde{T}_{1}$ in Theorem 1.2 can be improved if one knows that the growth rate of $f_{i}$ has either subexponential or polynomial.

Fix $A \geq 1, \gamma>1, \theta_{1}=10^{-6}$ and $\theta=\theta_{2} / 2=\theta_{1} / 10$ as before.

Theorem 5.1. Let $\delta \geq 0$ and $\varepsilon \in[0,1)$. If each $f_{i}$ is $(A, \gamma)$-regular on $\left[T_{1}, T_{2}\right)$ and satisfies

$$
f_{i}(t) \leq A e^{\delta t^{\varepsilon}} \quad \text { for all } t \in\left[T_{1}, T_{2}\right),
$$

then there exists a constant $C_{1}(A, \gamma, \delta, \varepsilon)>0$ such that for each $t \in\left[\widetilde{T}_{1}, T_{2}\right)$,

$$
\mathbb{P}_{x_{1}}\left(X_{t}=x_{2}\right) \leq \frac{C_{1}\left(v_{x_{2}} / v_{x_{1}}\right)^{1 / 2}}{\sqrt{f_{1}(t /(2 \gamma)) f_{2}(t /(2 \gamma))}} \exp \left(-\theta \frac{d_{\nu}\left(x_{1}, x_{2}\right)^{2}}{t}\right),
$$

where $\widetilde{T}_{1}=\left(2^{9} \delta T_{1}^{1+\varepsilon}\right) \vee d_{v}\left(x_{1}, x_{2}\right)$. 
Theorem 5.2. Let $\varepsilon \geq 0$. If each $f_{i}$ is $(A, \gamma)$-regular on $\left[T_{1}, T_{2}\right)$ and satisfies

$$
f_{i}(t) \leq A t^{\varepsilon} \quad \text { for all } t \in\left[T_{1}, T_{2}\right),
$$

then there exists a constant $C_{1}(A, \gamma, \varepsilon)>0$ such that (5.4) holds for $t \in\left[\widetilde{T}_{1}, T_{2}\right)$. Here,

$$
\widetilde{T}_{1}=\left(2^{10} \varepsilon T_{1} \log \left(T_{1} \vee 1\right)\right) \vee d_{v}\left(x_{1}, x_{2}\right) .
$$

Let's begin with Theorem 5.1. As the proof of Theorem 1.1, we need some results which are similar to Propositions 3.4 and 3.1.

Proposition 5.3. Let $\delta>0$ and $\varepsilon \in(0,1)$. Let $u, f$ be defined as in Section 3. Suppose further that $f$ is $(A, \gamma)$-regular on $\left[T_{1}, T_{2}\right)$ and satisfies

$$
f(t) \leq A e^{\delta t^{\varepsilon}} \quad \text { for all } t \in\left[T_{1}, T_{2}\right) .
$$

Then there exists a constant $C_{0}(A, \gamma, \delta, \varepsilon)>0$ such that for $R \geq \max \left\{4,2 \kappa^{(1+\varepsilon) /(1-\varepsilon)}, 2\left(\kappa T_{1}\right)^{(1+\varepsilon) / 2}\right\}$ and $t \in$ $\left[\kappa^{-1} R^{2 /(1+\varepsilon)}, T_{2} / 2\right)$, we have

$$
\left\langle u(t, \cdot)^{2}, 1-1_{B_{R}}\right\rangle \leq \frac{C_{0}}{f(t / \gamma)} \exp \left(-\theta_{1} \frac{R^{2}}{t}\right) .
$$

Here, $\kappa=(64 \delta)^{1 /(1+\varepsilon)}$.

Proof. We only show the part of the proof which is different from that of Proposition 3.4.

Fix $R \geq \max \left\{4,2 \kappa^{(1+\varepsilon) /(1-\varepsilon)}, 2\left(\kappa T_{1}\right)^{(1+\varepsilon) / 2}\right\}$ and $t \in\left[\kappa^{-1} R^{2 /(1+\varepsilon)}, T_{2} / 2\right)$. Take $L, D, \Delta, R_{j}$ and $t_{j}$ as in Proposition 3.4. We may still assume that $\theta_{1} \frac{R^{2}}{t}-L-\frac{1}{D \Delta} \geq \theta_{1}$. (Hence $t \leq R^{2}$ and $L \leq \theta_{1} \frac{R^{2}}{t}$.) However, we set

$$
j_{0}=\min \left\{j: R_{j}^{2 /(1+\varepsilon)} \geq \kappa t_{j}\right\} .
$$

Since $R \geq \max \left\{4,2 \kappa^{(1+\varepsilon) /(1-\varepsilon)}\right\}$, for $j<j_{0}$ we have

$$
t_{j}>\kappa^{-1} R_{j}^{2 /(1+\varepsilon)}>\kappa^{-1}(R / 2)^{2 /(1+\varepsilon)} \geq R / 2 \geq 2 .
$$

Hence

$$
t_{j}-t_{j+1}=t_{j} / 2 \geq R / 4=\Delta .
$$

From $R \geq 2\left(\kappa T_{1}\right)^{(1+\varepsilon) / 2}$, it deduces

$$
t_{j_{0}}=t_{j_{0}-1} / 2 \geq \kappa^{-1}(R / 2)^{2 /(1+\varepsilon)} / 2 \geq T_{1} / 2 .
$$

So, $T_{1} \leq 2 t_{j+1} \leq 2 t<T_{2}$ for each $j<j_{0}$. By the hypothesis that $f$ is $(A, \gamma)$-regular on $\left[T_{1}, T_{2}\right)$, one has

$$
f\left(2 t_{j+1}\right) \geq f(2 t) e^{-j L}
$$

the same as Lemma 3.2. Hence (3.8) holds under this circumstance, too. That is,

$$
\Lambda_{1}:=\sum_{j=1}^{j_{0}-1} \frac{\exp (j /(D \Delta))}{f\left(2 t_{j+1}\right)} \exp \left(-\frac{\left(R_{j}-R_{j+1}\right)^{2}}{D\left(t_{j}-t_{j+1}+\Delta\right)}\right) \leq \frac{e^{-\theta_{1}}\left(1-e^{-\theta_{1}}\right)^{-1}}{f(2 t)} \exp \left(-\theta_{1} \frac{R^{2}}{t}\right) .
$$

Next, if $t_{j_{0}}<R_{j_{0}}$ then by Proposition 2.7,

$$
\left\langle u\left(t_{j_{0}}, \cdot\right)^{2}, 1-1_{B_{R_{j_{0}}}}\right\rangle \leq c_{1} e^{-2 c_{1}^{-1} R_{j_{0}}} \leq c_{1} e^{-c_{1}^{-1} R} \leq c_{2} \exp \left(-\frac{\kappa}{16} R^{2 \varepsilon /(1+\varepsilon)}\right),
$$


where $c_{1}, c_{2} \geq 1$ are constants. If $t_{j_{0}} \geq R_{j_{0}}$ then by Proposition 2.7 we still have

$$
\left\langle u\left(t_{j_{0}}, \cdot\right)^{2}, 1-1_{B_{R_{j_{0}}}}\right\rangle \leq e^{-R_{j_{0}}^{2} /\left(8 t_{j_{0}}\right)} \leq \exp \left(-\frac{\kappa}{8} R_{j_{0}}^{2 \varepsilon /(1+\varepsilon)}\right) \leq c_{2} \exp \left(-\frac{\kappa}{16} R^{2 \varepsilon /(1+\varepsilon)}\right) .
$$

From $R_{j_{0}}^{2 /(1+\varepsilon)} \geq \kappa t_{j_{0}}$ and $R_{j_{0}-1}^{2 /(1+\varepsilon)}<\kappa t_{j_{0}-1}$, we get the following inequalities respectively:

$$
t_{j_{0}} \leq R^{2 /(1+\varepsilon)} / \kappa \quad \text { and } \quad j_{0}<\frac{1}{\log 2} \log \left(\frac{16 \kappa t}{R^{2 /(1+\varepsilon)}}\right) .
$$

Hence

$$
f(2 t) \leq f\left(2 t_{j_{0}}\right) e^{j_{0} L} \leq f\left(2 R^{2 /(1+\varepsilon)} / \kappa\right) \exp \left\{\frac{1}{\log 2} \log \left(\frac{16 \kappa t}{R^{2 /(1+\varepsilon)}}\right) \cdot L\right\}
$$

By (5.5) and the assumption $L \leq \theta_{1} \frac{R^{2}}{t}$,

$$
\begin{aligned}
f(2 t) & \leq A \exp \left(\frac{2^{\varepsilon} \delta}{\kappa^{\varepsilon}} R^{2 \varepsilon /(1+\varepsilon)}\right) \cdot \exp \left\{\frac{1}{\log 2} \log \left(\frac{16 \kappa t}{R^{2 /(1+\varepsilon)}}\right) \cdot \theta_{1} \frac{R^{2}}{t}\right\} \\
& \leq A \exp \left(\frac{2^{\varepsilon} \delta}{\kappa^{\varepsilon}} R^{2 \varepsilon /(1+\varepsilon)}\right) \exp \left\{\frac{\theta_{1}}{e \log 2} 16 \kappa R^{2 \varepsilon /(1+\varepsilon)}\right\} .
\end{aligned}
$$

Since $t \leq R^{2}$ and $R \geq 4$, there exists a constant $c_{3}$ such that

$$
\begin{aligned}
\exp \left(\frac{j_{0}}{D \Delta}\right) & \leq \exp \left(\frac{1}{\log 2} \log \left(\frac{16 \kappa t}{R^{2 /(1+\varepsilon)}}\right) \cdot \frac{1}{25 R}\right) \\
& \leq \exp \left(\frac{1}{\log 2} \log \left(\frac{16 \kappa R^{2}}{R^{2 /(1+\varepsilon)}}\right) \cdot \frac{1}{25 R}\right) \leq c_{3} .
\end{aligned}
$$

Combining the above inequalities gives

$$
\begin{aligned}
\Lambda_{2} & :=\exp \left(\frac{j_{0}-1}{D \Delta}\right)\left\langle u\left(t_{j_{0}}, \cdot\right)^{2}, 1-1_{B_{R_{j_{0}}}}\right\rangle \\
& \leq c_{3} c_{2} \exp \left(-\frac{\kappa}{16} R^{2 \varepsilon /(1+\varepsilon)}\right) \\
& \leq c_{3} c_{2} \exp \left(-\frac{\kappa}{16} R^{2 \varepsilon /(1+\varepsilon)}\right) \cdot \frac{1}{f(2 t)} \cdot A \exp \left(\frac{2^{\varepsilon} \delta}{\kappa^{\varepsilon}} R^{2 \varepsilon /(1+\varepsilon)}\right) \exp \left\{\frac{\theta_{1}}{e \log 2} 16 \kappa R^{2 \varepsilon /(1+\varepsilon)}\right\} \\
& =\frac{c_{4}}{f(2 t)} \exp \left(\left(-\frac{\kappa}{16}+\frac{2^{\varepsilon} \delta}{\kappa^{\varepsilon}}+\frac{\theta_{1}}{e \log 2} 16 \kappa\right) R^{2 \varepsilon /(1+\varepsilon)}\right),
\end{aligned}
$$

where $c_{4}=c_{3} c_{2} A$. Substituting $\kappa=(64 \delta)^{1 /(1+\varepsilon)}$ and using the condition $t \geq \kappa^{-1} R^{2 /(1+\varepsilon)}$,

$$
\begin{aligned}
\Lambda_{2} & \leq \frac{c_{4}}{f(2 t)} \exp \left(\left(-\frac{\kappa}{16}+\frac{2^{\varepsilon} \kappa}{64}+\frac{\theta_{1}}{e \log 2} 16 \kappa\right) R^{2 \varepsilon /(1+\varepsilon)}\right) \\
& \leq \frac{c_{4}}{f(2 t)} \exp \left(-\frac{\kappa}{64} R^{2 \varepsilon /(1+\varepsilon)}\right) \\
& \leq \frac{c_{4}}{f(2 t)} \exp \left(-\frac{1}{64} \frac{R^{2}}{t}\right) .
\end{aligned}
$$

This completes the proof. 
Proposition 5.4. Under the condition of Proposition 5.3, there exists a constant $C_{0}(A, \gamma, \delta, \varepsilon)>0$ such that

$$
\left\langle u(t, \cdot)^{2}, \exp \left(\theta_{2} \frac{\left(d_{\nu}(o, \cdot) \wedge(2 t)\right)^{2}}{t}\right)\right\rangle \leq \frac{C_{0}}{f(t / \gamma)} \quad \text { for } t \in\left[2^{8} \delta T_{1}^{1+\varepsilon}, T_{2} / 2\right) .
$$

Proof. We only show the difference from Proposition 3.1. Fix $\kappa=(64 \delta)^{1 /(1+\varepsilon)}$ and $t_{0}=\max \left\{16,4 \kappa^{(2+2 \varepsilon) /(1-\varepsilon)}\right.$, $\left.\kappa^{-(1+\varepsilon) / \varepsilon}\right\}$. Let $t \in\left[2^{8} \delta T_{1}^{1+\varepsilon}, T_{2} / 2\right)$. If $t \leq t_{0}$, then as before the result is trivial. So, we may assume further $t \geq t_{0}$. Fix $R=t^{1 / 2}$. Then

$$
R \geq \max \left\{4,2 \kappa^{(1+\varepsilon) /(1-\varepsilon)}, 2\left(\kappa T_{1}\right)^{(1+\varepsilon) / 2}\right\} \quad \text { and } \quad \kappa t \geq R^{2 /(1+\varepsilon)} .
$$

Define $\theta_{2}, \Upsilon_{j}$ and $n$ as in Proposition 3.1. However, we set

$$
m=\max \left\{j: \kappa t \geq\left(2^{j} R\right)^{2 /(1+\varepsilon)}\right\} .
$$

Then by Proposition 5.3, for $1 \leq j \leq m \wedge n$ we have

$$
\Upsilon_{j} \leq \frac{C_{0}}{f(t / \gamma)} \exp \left(-\theta_{2} \cdot 4^{j-1}\right)
$$

If $m+1 \leq j \leq n$ then use Proposition 2.7 and get

$$
\Upsilon_{j} \leq e^{4^{j} \theta_{2}}\left\langle u(t, \cdot)^{2}, 1-B_{2^{j-1} R}\right\rangle \leq e^{4^{j} \theta_{2}} \cdot \exp \left(-\frac{\left(2^{j-1}\right)^{2}}{8}\right) \leq \exp \left(-\frac{4^{j-1}}{12}\right) .
$$

By the definition of $m$, one has $\kappa t<\left(2^{m+1} R\right)^{2 /(1+\varepsilon)}=\left(2^{m+1} t^{1 / 2}\right)^{2 /(1+\varepsilon)}$ and so,

$$
4^{m+1}>\kappa^{1+\varepsilon} t^{\varepsilon}=64 \delta t^{\varepsilon} .
$$

By (5.5) and (5.6), we still have

$$
\begin{aligned}
\Upsilon_{j} & \leq \frac{A e^{\delta t^{\varepsilon}}}{f(t)} \exp \left(-\frac{4^{j-1}}{12}\right) \leq \frac{A}{f(t)} \exp \left(4^{m-2}-\frac{4^{j-1}}{12}\right) \\
& \leq \frac{A}{f(t)} \exp \left(4^{j-3}-\frac{4^{j-1}}{12}\right)=\frac{A}{f(t)} \exp \left(-\frac{4^{j-1}}{48}\right) .
\end{aligned}
$$

For the other terms $\Upsilon_{0}$ and $\Upsilon_{\infty}$, one can get the estimates the same as we did in Proposition 3.1 and so we finish the proof.

Proof of Theorem 5.1. If $\varepsilon \delta=0$, then the problem is reduced to Corollary 2.8 since each $f_{i}$ has a constant upper bound on $\left[T_{1}, T_{2}\right)$. Otherwise, if $\delta>0$ and $\varepsilon \in(0,1)$ then we can get the proof as Theorem 1.1 by using Proposition 5.4 and the Cauchy-Schwarz inequality.

Proof of Theorem 5.2. We obtain a similar result as Proposition 5.3 just by setting

$$
j_{0}=\min \left\{j: R_{j}^{2} / \log R_{j} \geq \kappa t_{j}\right\},
$$

and then prove the theorem as above.

Enlightened by Boukhadra, Kumagai and Mathieu [6], we give an application of Theorem 5.1. (See our further reseach [7] for the application in a concrete example.) Set

$$
p_{t}(x, y)=\frac{\mathbb{P}_{x}\left(X_{t}=y\right)}{v_{y}}
$$

for the heat kernel of $X$. 
Example 5.5. Suppose $p_{t}\left(x_{i}, x_{i}\right) \leq \kappa t^{-d / 2}$ for $t \geq t_{1}$ and $i \in\{1,2\}$. Suppose $v_{x_{1}}, v_{x_{2}} \geq t_{1}^{-\kappa}$. Then for each $\varepsilon>0$, there exists a constant $C_{0}(d, \kappa, \varepsilon)>0$ such that

$$
p_{t}\left(x_{1}, x_{2}\right) \leq C_{0} t^{-d / 2} \exp \left(-\theta \frac{d_{\nu}\left(x_{1}, x_{2}\right)^{2}}{t}\right) \quad \text { for } t \geq t_{1}^{1+\varepsilon} \vee d_{\nu}\left(x_{1}, x_{2}\right)
$$

Proof. Let $f_{i}(t)=\kappa^{-1} v_{x_{i}}^{-1} t^{d / 2}$ for $i \in\{1,2\}$ and $t \in \mathbb{R}_{+}$. Then for each $t \geq t_{1}$,

$$
\mathbb{P}_{x_{i}}\left(X_{t}=x_{i}\right)=v_{x_{i}} p_{t}\left(x_{i}, x_{i}\right) \leq \frac{1}{f_{i}(t)} .
$$

Note that $f_{i}$ is $(1,2)$-regular and for $t \geq t_{1}$,

$$
f_{i}(t)=\kappa^{-1} v_{x_{i}}^{-1} t^{d / 2} \leq \kappa^{-1} t_{1}^{\kappa} t^{d / 2} \leq \kappa^{-1} t^{\kappa+d / 2} \leq A \exp \left(2^{-9} t^{\varepsilon}\right),
$$

where $A$ is some constant which depends only on $d, \kappa$ and $\varepsilon$. Applying Theorem 5.1 gives

$$
\mathbb{P}_{x_{1}}\left(X_{t}=x_{2}\right) \leq \frac{C_{1}\left(v_{x_{2}} / v_{x_{1}}\right)^{1 / 2}}{\sqrt{f_{1}(t / 4) f_{2}(t / 4)}} \exp \left(-\theta \frac{d_{v}\left(x_{1}, x_{2}\right)^{2}}{t}\right) \quad \text { for } t \geq t_{1}^{1+\varepsilon} \vee d_{v}\left(x_{1}, x_{2}\right),
$$

which implies (5.7) immediately.

\section{Acknowledgements}

I am greatly indebted to Martin Barlow for suggesting the problem and for many stimulating conversations. I gratefully acknowledge the valuable comments and discussions with Minzhi Zhao of Zhejiang University. The research was partially supported by the NSFC grants (No. 11001173 and No. 11531001), and China Scholarship Council during the author's visit to University of British Columbia in 2012.

\section{References}

[1] S. Andres, M. T. Barlow, J. D. Deuschel and B. M. Hambly. Invariance principle for the random conductance model. Probab. Theory Related Fields 156 (2013) 535-580. MR3078279

[2] M. T. Barlow. Random walk on supercritical percolation clusters. Ann. Probab. 32 (2004) 3024-3084. MR2094438

[3] M. Barlow and X. Chen. Gaussian bounds and parabolic Harnack inequality on locally irregular graphs. Preprint, 2013. Available at http:// www.math.ubc.ca/ barlow/preprints/107_gb_irreg.pdf.

[4] N. Berger, M. Biskup, C. E. Hoffman and G. Kozma. Anomalous heat-kernel decay for random walk among bounded random conductances. Ann. Inst. Henri Poincaré Probab. Stat. 44 (2008) 374-392. MR2446329

[5] M. Biskup and T. M. Prescott. Functional CLT for random walk among bounded random conductances. Electron. J. Probab. 12 (2007) 1323-1348. MR2354160

[6] O. Boukhadra, T. Kumagai and P. Mathieu. Harnack inequalities and local central limit theorem for the polynomial lower tail random conductance model. J. Math. Soc. Japan 67 (2015) 1413-1448. MR3417502

[7] X. Chen. Gaussian bounds and collisions of variable speed random walks on lattices with power law conductances. Available at arXiv: $1507.05297 \mathrm{v} 2$.

[8] T. Coulhon, A. Grigor'yan and F. Zucca. The discrete integral maximum principle and its applications. Tohoku Math. J. 57 (2005) $559-587$. MR2203547

[9] E. B. Davies. Analysis on graphs and noncommutative geometry. J. Funct. Anal. 111 (1993) 398-430. MR1203460

[10] E. B. Davies. Large deviations for heat kernels on graphs. J. London Math. Soc. 47 (1993) 65-72. MR1200978

[11] M. Folz. Gaussian upper bounds for heat kernels of continuous time simple random walks. Electron. J. Probab. 16 (2011) $1693-1722$. MR2835251

[12] M. Folz. Volume growth and stochastic completeness of graphs. Trans. Amer. Math. Soc. 366 (2014) 2089-2119. MR3152724

[13] R. L. Frank, D. Lenz and D. Wingert. Intrinsic metrics for non-local symmetric Dirichlet forms and applications to spectral theory. J. Funct. Anal. 266 (2014) 4765-4808. MR3177322

[14] A. Grigor'yan. Gaussian upper bounds for heat kernel on arbitrary manifolds. J. Differential Geom. 45 (1997) 33-52. MR1443330

[15] W. Hebisch and L. Saloff-Coste. Gaussian estimates for Markov chains and random walks on groups. Ann. Probab. 21 (1993) 673-709. MR1217561

[16] P. Mathieu and E. Remy. Isoperimetry and heat kernel decay on percolation clusters. Ann. Probab. 32 (2004) 100-128. MR2040777

[17] K. T. Sturm. Analysis on local Dirichlet spaces I. Recurrence, conservativeness and $L^{p}$-Liouville properties. J. Reine Angew. Math. 456 (1994) 173-196. MR1301456 